\section{Hands-on brushing}

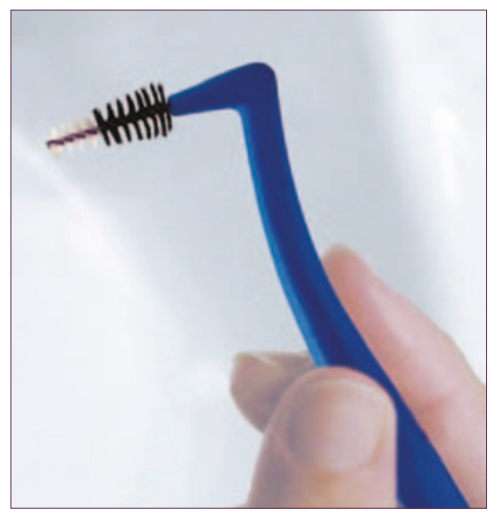

Do your patients have difficulty accessing and getting between all of their teeth? INTERPROX PLUS has designed a brush with a fixed angle and a longer handle than traditional hand held brushes to reach every area of the mouth. Even patients with large hands should find effective brushing effortless.

INTERPROX

PLUS

brushes also have half black bristles - to show effective plaque removal - and half white bristles so that patients can monitor and improve their bleeding score. INTERPROX say that the bristles, which come with a protective cap, are high quality and will maintain their shape after repeated use.

A range of sizes are available: mini, micro and conical and can be purchased in boxes of 100 single packets and also blister packs of six brushes. The singles enable patients to choose different sizes as required.

Special offer in March and April 2006: buy a dozen blister packs and get a dozen single packets free. Freephone 08009801517 for a free trial pack. For more information call 02084597550.

\section{The perfect filaments}

Many toothbrushes are too hard and too thick, resulting in two critical oral care problems: they cannot remove plaque because they cannot reach the gum line, and the pressure necessary to direct bristles into a niche may damage gums or abrade tooth enamel.

Curaprox claims to have found a solution to these problems by designing gentle, deep-cleaning CUREN ${ }^{\circledR}$ bristles. These filaments absorb water six times slower than nylon bristles, enabling them to keep their original texture even when wet. This makes the use of much finer filaments possible, which deliver gentler and more effective cleaning. Another feature of CUREN ${ }^{\circledR}$ bristles is that

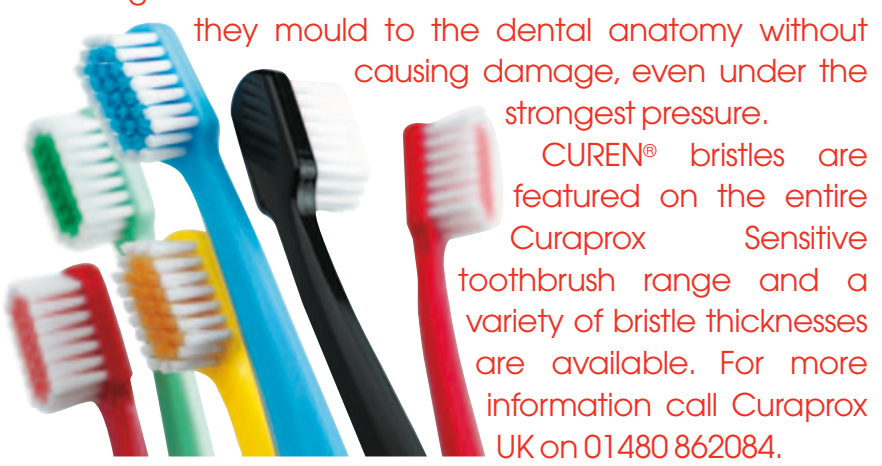

\section{New mouthwash range}

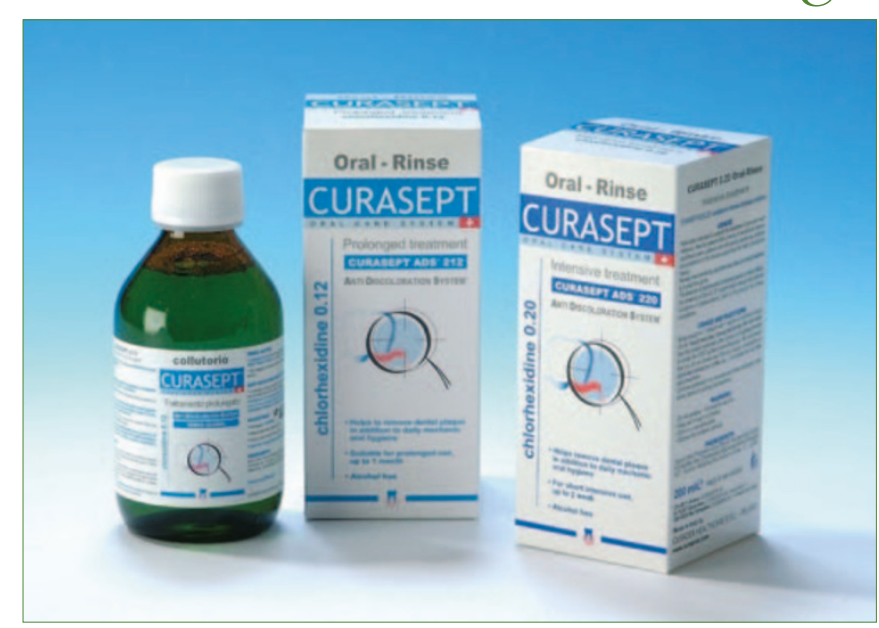

Curasept ADS, developed by Curaprox, is a new range of alcohol-free mouthwashes with a difference. Curasept offers all the benefits of a chlorhexidine mouthwash but has been developed to minimise the effects commonly associated with chlorhexidine, such as oral discolouration and changes in taste perception. It therefore offers patients an alternative to traditional chlorhexidine mouthwashes.

Intended for short-term intensive use, Curasept can be used either before or after treatment, including endodontic and implant procedures, and helps to prevent post-treatment complications when used as advised. It is also effective against plaque and the development of caries.

For more information contact Curaprox UK on 01480862084.

\section{Stylish workwear}

Alexandra plc has launched a new-look, bigger catalogue to showcase its corporate uniforms and workwear.

The healthcare range leads with a technologically advanced range of

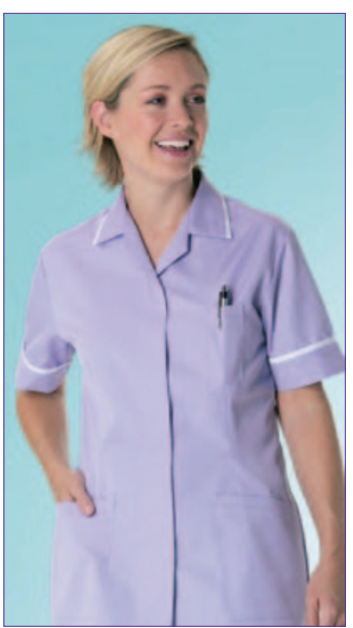
clothing which has been developed to help reduce the risk of cross-infection. The clothing is manufactured in an advanced protectivewear fabric, Bioguard, which is specifically designed to give long-lasting antimicrobial protection. Alexandra has also added new colours to key styles for increased choice and flexibility.

The company also has an online shop, which features full product details for all 900 styles, live stock information and quick online registration, as well as special offers. For more information visit www.alexandra.co.uk or phone 08700600200 . 